\title{
Grinding of the gears with high depth processing
}

\author{
Sergiu Mazuru, Serghei Scaticailov, and Ion Stingaci* \\ Technical University of Moldova, 9, Studenţilor str. Chişinău, Republic of Moldova
}

\begin{abstract}
The goal research consists in the analysis and synthesis of technological schemes, processes, tools and equipment for processing of conical gears and precession gears with medium module. A new processing method which allows to intensify the process of processing as a result is proposed. It ensures a required accuracy of processing. The theoretical researches are based on the theory gear drives, theory of involute profiles generation and precession gears, generating machine, tool design theory machine building technology. Methods of mathematical analysis, analytic geometry, mathematical model and 3D computer model are used. The experimental researches were conducted in laboratories of departments of machine manufacturing technology and fundamentals of machines design in the Technical University of Moldova, by using machines and technological equipment for machining and testing. Experimental data processing were accomplished using approximation methods and mathematical statistics.
\end{abstract}

\section{Background of profile deep grinding of gear elements}

By its kinematic, the deep grinding differs substantially from traditional grinding methods and in particular from pendular processing scheme. Larger material layer thickness - up to $10 \mathrm{~mm}$ or more, removable in a single pass, in conjunction with low workpiece feed - up to $20-40 \mathrm{~mm} / \mathrm{min}$, leads to the fact that the contact length of the abrasive tool with a workpiece and the lengths of the zones characterized by thermodynamic effects are one two orders higher than those corresponding to traditional forms of pendular grinding.

The characteristics of deep grinding gears made of hardened steel deep grinding, due to the exact shape and large size of the contact area of the grinding wheel with the surface to be processed and its elevated sensitivity to thermal injury involves the unconditional fulfilment of the following requirements:

a)during grinding process, the cavity between the adjacent tines machined in a single pass must provide a high efficiency of material removal, this being of $3-4$ times higher than, for example, creep feed grinding of the blade roots made of nickel alloys;

b) the maximum allowable temperature in the cutting zone with respect to time of one deep grinding cavity should be about $1.5-2$ times less than that corresponding to the creep feed grinding, applied to heat-resistant nickel and titanium alloys;

\footnotetext{
* Corresponding author: ion.stingaci@gmail.com
} 
c) the need to maintain the original profile of the grinding wheel while processing a maximum number of gears and multiple cavities in the period between revisions, as used in gear grinding machines, with just cyclical corrections.

Thus, effective implementation of profiled deep grinding process is possible, it ensuring a high productivity with minimum concomitant heating temperature $\left(700^{\circ} \mathrm{C}\right)$. Conditions of forming the cavity found between two teeth in one or more passes with the grinding wheel are determined mainly by its cross-sectional area and the size of the contact surfaces between tool and workpiece [1-6].

The problem of finishing conical gears was a preoccupation for the researchers in the field of manufacturing technologies. Thus, Lopatin and Plotnikova investigated the possibilities of positioning the tools and the workpiece in the case of using shaving process and grinding process to finish helical bevel gears teeth flanks [7]. They noticed that the common devices used for obtaining teeth or devices similar to those could be also applied in the finishing processes.

Komatsubara et al. studied the concave conical gear used in marine transmissions. They took into consideration a theoretical method of generating the concave conical gears, aiming to establish the essential dimensions of gear and tool [8]. The experiments proved a good correspondence of the principal normal radii of test gears with the values theoretically determined. Another remark was that the Hertzian contact ellipse generated in the case of concave conical gears is larger than that corresponding to the conventional conical gear.

The problem of tooth contact analysis in the case of conical involute gears was investigated by Jingliang et al. [9]. They developed a mathematical model corresponding to the conical involute gears, considering the theory of gearing and the generating mechanism.

Various aspects concerning the conical gears and methods for their machining were investigated by Radzevich [10]. The use of form grinding wheel is discussed, but especially in the case of grinding cylindrical gears.

The objective of this paper was to highlight some aspects corresponding to the deep grinding process applied in the case of conical gears, by considering the conditions specific to these gears.

When optimizing the parameters of the of creep feed grinding mode range $\mathrm{Vk}$, the speed of longitudinal movement of the workpiece or grinding wheel and the cutting depth are considered; the main difficulty is to solve the problems of single -pass profiling, and imposing cuts at multipass profiling gear wheels. Figure 1 shows the schema of gears deep profiled grinding.

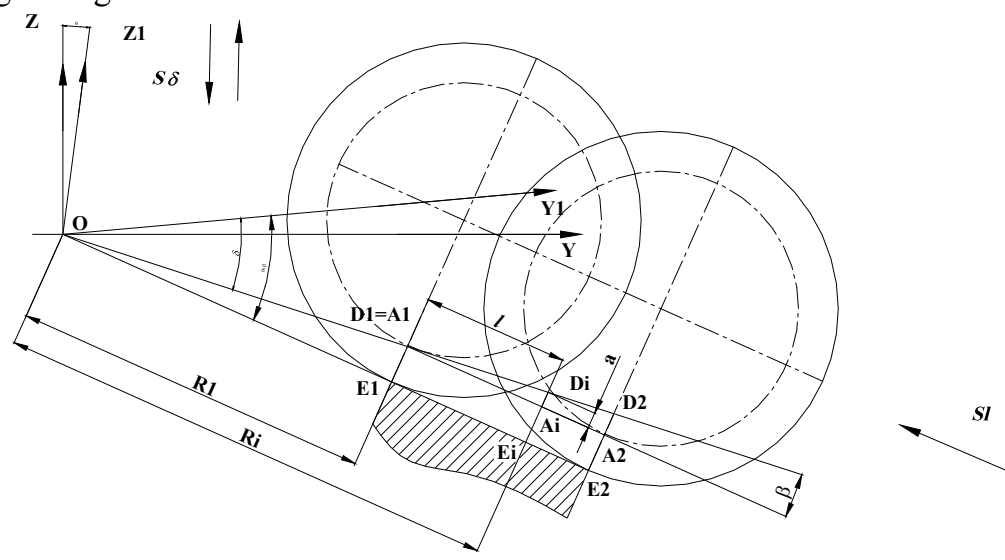

Fig. 1. The deep profile grinding of gear.

The most important kinematic parameter of the process is the area of cavity section $F_{c}$, which is affected by the tool movement in a single pass. $F_{c}$ depends on the size of the 
workpiece material cutting resistance, which is proportional to the cross sectional area of the shear layer, and accordingly, to the intensity of the thermodynamic tension in the grinding zone. In addition, the workpiece material determines the performance of the profiling process speed during the material removal.

$$
W=F_{c} \cdot S_{d}
$$

Analysis of calculation of cavity section area formed in a single pass shown that $F_{c}$ section increases with the number of the teeth $z$. The extent of this effect is negligible: with unlimited including of the teeth, the decrease of the $F_{c}$ is only of $5.7 \%$. This small difference makes possible the analysis of the process of wheels formation, in a first approximation, without taking into account the number of teeth.

In forming cavity between the adjacent teeth in a single pass, the sectional area of $F_{c}$ increases from 14.95 to $229.4 \mathrm{~mm}^{2}$, this meaning of about 15.3 times.

Accordingly, the cutting resistance increases, this being necessary to be considered when evaluating the possibility of profiling gears with large modules in a single pass.

The creep feed grinding for the first pass of the grinding wheel is advisable to appoint a cutting depth, which will provide the final shapes of the teeth, or taking into account the retained allowance for finish grinding after thermal or chemical-thermal treatment. However, this option is largely due to the technical capabilities of the machine and tools, as well as to the restrictions derived from the thermodynamic conditions of the current process without forming defects.

As an example, one can see the way of changing the sectional area $F_{c}$ depending on the teeth height $h$ and cutting depth of the first pass $t$ (Figure 2).

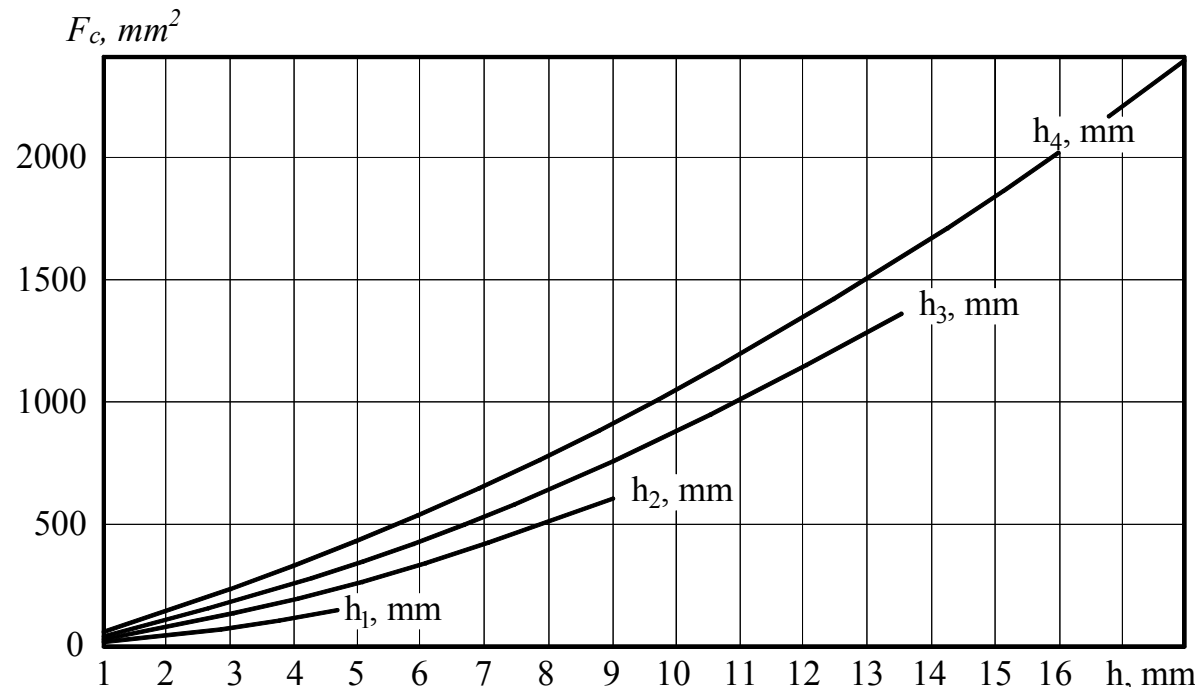

Fig. 2. Changing the cross sectional area slice $F_{c}$, depending on the height of the teeth $h$ and the depth grinding.

In its turn, the required power shaping as thermodynamic processing quality conditions depends not only on the component due to the removed slice section $F_{c}$, but on the friction work, able to contribute to the overall grinding energy consumption and which could be quite noticeable.

Frictional work is proportional to the contact area of the grinding wheel with $F_{k}$ treated surface, which for the deep grinding of gear is determined by the scheme shown in Figure 1. 
The total area of the contact zone of the friction develops along the bottom cavity width and with a length of the contact $s$, equal to the contact arc $\mathrm{AB}$, and two inclined side surfaces, the dimensions of which depend on the ratio of the width $B$ and the length AC. Figure 3 shows the change in cross-sectional area $F_{c}$ cavity and $F_{k}$ contact area of the grinding wheel diameter $D=85 \mathrm{~mm}$. Let us note that the estimated value of $F_{k}$ significantly

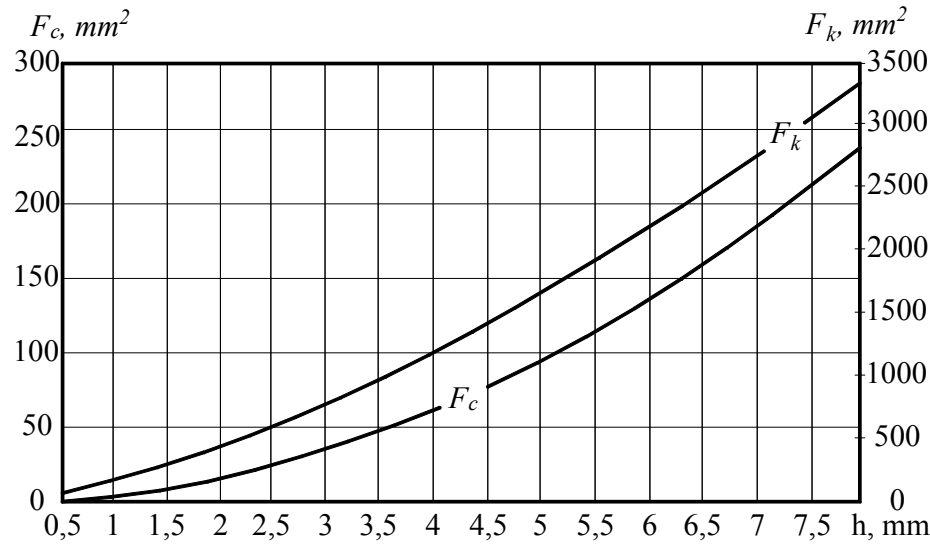

Fig. 3. Comparison of the calculated values of the area $F_{c}$ the slice section and $F_{k}$ contact with the grinding gear wheels

exceeds the pre-sectional area $F_{c}: h 1$ at $F_{k}$ value is greater than $F_{c}$ of about 55.4 times, while the excess is stored h4 great, but is reduced up to 13.8 times.

The most closely linked feed rate and cross-sectional area (the simple correlation coefficient is of 0.838 ), as well as the grinding speed, weakly correlated with each other kinematic and other operating parameters of the process, indicating their statistical independence.

Thus, we can talk about the decisive influence exerted by work profile creep feed grinding gear wheels on the parameter assignment of movement speeds. It is appropriate to stress that, in turn, is a function of the magnitude of the cutting depth, of the module and number of the teeth of the gear being processed.

The stability of the thermodynamic effects on the machined gears, due to dispersion conditions with vigorous rubbing, has, as shown by the results of the study, a decisive influence on the quality and accuracy of the profiled of the teeth.

From the analysis of the experimental data, one notices that it is important in relation to pragmatic conclusion: to stabilize the thermodynamic effect of processing of the gear, it is necessary to stabilize any controlled input parameter shaping process.

Such a parameter can be the value of the removed section of cavity, which is regulated by the depth of the passage. In conclusion, it should be noted that on the basis of theoretical analysis and preliminary experimental studies, there was established a fundamental possibility of implementing the process of forming gear wheels profile by creep feed grinding method.

The methodology for optimizing profiling in one or more passes of the grinding wheel depends on the characteristics of the toothed wheel and its handling process conditions.

It is also important to emphasize that the fundamental difference between the new process of forming gear wheels known as an analogy of profile creep feed grinding is that it provides a high performance material removal at a lower heating of surface layer to be treated to prevent the appearance of sanding defects.

Kinematic forming of cavity implies an increased thermodynamic process intensity with an intense heating. The need to obtain an accurate profile and the minimum accumulated 
pitch error of machined wheels with circular grinding wheel dressing imposes stringent requirements for its durability and evenness of wear resistance for the service period.

Under these requirements, it is advisable to use high hardness tool that becomes an additional factor able to intensify the heating process. In this connection, the abrasive tool for profile creep feed grinding gear wheels have special requirements that are to be the optimum combination of necessary values of its strength and durability, together with very high cutting properties, able to minimize the stresses generated by the thermodynamic process.

When profile creep feed grinding, to avoid overheating of the workpiece material, usually the soft abrasive tools with abundant cooling of the cutting area are used.

To ensure high accuracy to the formed profile, tool is subject to continuous compulsory revision with special diamond profiled rollers. In this case, when using the cyclic changes, it is possible to use the tools with higher hardness and which should be reformed quickly and easily to the desired cavity profile of machined gears.

The problem in grinding is mainly the work of friction of the abrasive grains on the surface to be processed, the weight of which is up to $70-80 \%$ of the total energy costs for material removal. This is due to the fact that most of the abrasive grains, which are at the working surface of the grinding wheel do not participate in the cutting process, and only deform (crushes) the processed material, being a significant source of heat during grinding. The abrasive tool is the determining element in the process of grinding system.

The correct assignment of the grinding wheel characteristics largely depend on character of the grinding process, the level of performance, the intensity of heating, tool life, machining accuracy and quality.

\section{Assigning characteristics highly porous wheel for gear grinding}

In the grinding interaction of each abrasive grain and a grinding wheel as a whole with the cutting material takes place under extreme conditions. Under the high pressures and temperatures in the cutting zone the adhesive grasp the abrasive with the work material, the mutual diffusion of chemical elements, increase work bundle friction and abrasive grains of the grinding wheel with the workpiece surface and, as a consequence - the softening and breakdown of the abrasive grains and the formation of the surface layer parts with unfavorable conditions for the operation of the properties. Figure 1 shows a block diagram of the relationship of the constituent elements of the grinding wheel - grinding grain and bundle it with properties that need to be considered when appointing the tool characteristics. Under these conditions, complex thermodynamic efficiency of the grinding wheel is determined by the ability of abrasive grains on the working surface of the tool to resist said contact physicochemical phenomena and, above all, their softening and thermal degradation, as well as the ability to bundle hold the abrasive grains to the loss of cutting properties. In accordance with the block diagram, you can define the characteristics of grinding wheels for gear grinding optimal conditions. These include: a) optimal assignment modification of alumina as an abrasive material under high temperatures and high resistance to brittle fracture; b) grit selection range to provide the desired grinding performance with a minimum roughness of the processed surface; c) to find the optimal ratio of the structure (porosity) of the grinding wheel and its hardness to ensure a minimum intensity of heat and maximum durability of the tool; d) selection of a ceramic bond, which firmly holds the abrasive grains under conditions of intense heat generation at a minimum of friction with the workpiece surface. 


\section{Conclusion}

The theoretic al analysis showed that the main technical difficulty of implementing profiling gearwheels grinding process is extremely unfavorable combination of large sections of the removed material Fc with more than an order of magnitude of larger contact area Fk wheel with machined surface profile. Dimensions Fc and Fk determine respectively the two main characteristic components of the cutting operation: removal of the workpiece material and the work of the friction. Both kinematic components responsible for the thermodynamic strength of the cutting process and the stability of its course are very dependent on the gear unit element $\mathrm{m}$ and the number of passes.

The optimal choice of the characteristics of the grinding wheel provides the related appointment of grain, hardness and structure of the instrument based on unconditional restrictions to ensure the set parameters on material removal rate, the roughness of the treated surface of the part and the thermal stress in the cutting zone. Optimization of other characteristics of a highly porous material on the tool abrasive grain, hardness and operational properties ligament takes into account, in addition to these restrictions, the need for increased durability and efficiency of the grinding process.

If you have been on the working surface of the grinding wheel, which can collect the chips, increased cutting power tool, as it increases the time may come when his "brining", that is, the loss of cutting properties by filling the pores chips. In addition, the selection of the optimal ratio of "grain-hardness-structure" by optimizing the composition of the abrasive mass and ceramic binder can provide grinding conditions for grinding wheel wear process takes place in a self-sharpening mode.

\section{References}

1. I. Bostan, S. Mazuru, S. Scaticailov, TEHNOMUS Journal 20 (2013)

2. S. Mazuru, A. Vlase and S. Scaticailov, Processing technologies on machine tools for machining gears (in Romanian) (Tehnica-U.T.M Publishing House, Chişinău, 2014))

3. I. Bostan, S. Mazuru, M. Vaculenco, S. Scaticailov, Proceedings of the IX International Congress "Machines, Technologies, Materials 2012”, (Varna, Bulgaria, 2012)

4. V. Botnari and S. Mazuru, Applied Mechanics and Materials 657 (2014)

5. S. Mazuru S. and M. Casian, Advanced Materials Research 1036 (2014).

6. M. Casian and S. Mazuru, Advanced Materials Research 1036 (2014)

7. B.A. Lopatin, S.V. Plotnikova, Procedia Engineering, 150 (2016)

8. H. Komatsubara, K. Mitome, T. Ohmachi, JSME International Journal 452 (2002)

9. HE Jingliang, WU Xutang, CUI Yahui, NIE Gang, Chinese Journal Of Mechanical Engineering 191 (2006)

10. S.P. Radzevich, Gear cutting tools. Fundamentals of design and computation (Taylor and Francis Group, Broken Sound Parkway, U.S.A., 2010) 